\title{
Use of budesonide in severe asthmatics aged
} 1-3 years

\author{
Gary J Connett, Catherine Warde, Edwina Wooler, Warren Lenney
}

\begin{abstract}
The treatment of severe asthmatics aged 1-3 years with budesonide administered via Nebuhaler and Laerdal facemask was assessed in a six month, double blind, placebo controlled study.

Patients were randomised to budesonide $(n=20)$ or placebo $(n=20)$ treatment. Daily symptom scores were recorded by parents and clinic visits occurred every six weeks.

Cough scores improved significantly with budesonide: nocturnal and daytime cough scores (0-2) fell by a mean (SEM) of $0.4(0.2)$ and $0.5(0.2)$; while with placebo the nocturnal score increased by $0.1(0 \cdot 2)$ and daytime score was unchanged at 0.0 $(0 \cdot 2)$. All other data showed improvements for active over placebo treatment. Thirteen children in each group completed six months' treatment. Parents of 25 of these found the delivery system convenient and easy to use. While blinded to treatment it was assessed to be beneficial in nine of 13 receiving budesonide and four of 13 receiving placebo.

The Nebuhaler and facemask is an acceptable delivery system for long term asthma treatment. Symptoms are significantly reduced when it is used to give 400-800 $\mu \mathrm{g}$ budesonide/day to children aged 1-3 years who cannot use other delivery systems.

(Arch Dis Child 1993; 69: 351-355)
\end{abstract}

Recurrent respiratory symptoms are common in early childhood. In recent years, it has been recognised that those with frequent and severe symptoms often have asthma persisting into later childhood. ${ }^{1}$ With increased awareness of the importance of inflammation in the pathogenesis of asthma, ${ }^{2}$ the use of anti-inflammatory treatment early in the course of the disease might be an opportunity to minimise lung damage and attempt to improve the natural history of what can become a debilitating illness.

This study was designed to provide data on the optimal long term use of steroids in young children with significant morbidity from asthma, using currently available knowledge about steroid usage in this age group. Some studies using oral prednisolone to treat acute symptoms have been disappointing in children under 2 years, ${ }^{34}$ but effective in children over 2 years. ${ }^{4}$ One study has suggested that intramuscular methylprednisolone is also effective. ${ }^{5}$ More recently, there have been favourable reports on the use of inhaled steroid prophylaxis using a spacer device and facemask. ${ }^{6-8}$ These fast and simple delivery systems are an attractive alternative to nebulisers and reduce the possibility of side effects that occur with systemic treatment.

It is recognised that different aetiological factors underlie common respiratory symptoms characteristic of asthma in this age group. Factors such as airway size, previous bronchiolitic illness, and the presence of atopy, have different prognostic implications and might account for the conflicting reports on the effects of nebulised steroids in the first year of life, a time when there is the greatest diagnostic uncertainty. ${ }^{9-11}$ Coughing is a particularly prominent symptom in children with persistent asthma, ${ }^{12}$ although its relationship to wheeze is unclear. ${ }^{13}$ Objective measurements in school age children suggest that night time cough is less frequent than that suggested by parents, ${ }^{14}$ but in younger children it is often reported as the most troublesome symptom and is disruptive to family life; bronchodilators are often ineffective. ${ }^{15}$

This study investigates the use of budesonide (Pulmicort, Astra), delivered via a large volume spacer (Nebuhaler, Astra) and Laerdal facemask, in children aged 1-3 years with persistent asthma symptoms including chronic cough. Parallel groups receiving active or placebo treatment were studied for up to six months to assess the long term effectiveness and acceptability of this treatment in children who were unable to use the mouthpiece of a spacer. Efficacy was measured in terms of symptoms, effects on the lifestyle of the children and parents, and the use of medication for asthma.

\section{Patients and methods}

Children recruited were hospital referrals aged 1-3 years, with at least a six month history of troublesome asthma and thought by their parents to be responsive to bronchodilators. Chest radiographs were taken in an attempt to exclude other causes of wheezing. Entry into the two week run-in period was conditional to no clinical evidence of respiratory tract infection and no treatment with inhaled or oral steroids in the previous fortnight. Parents recorded daily symptom scores and gave their children one puff twice a day of placebo medication through a large volume spacer and Laerdal facemask (size 02).

They were told to shake the canister and release one dose of aerosol into the Nebuhaler chamber held horizontally with the valve closed. They were then told to hold the mask of the Nebuhaler over their child's face and to 
try and keep it in place for up to 20 seconds. The chamber was tilted to at least $30^{\circ}$ so that the valve fell open, and if possible a seal was made between the mask and the child's face. Valve closure with expiration was not essential, although some children achieved it. Some parents found it easier to give twice daily medication to their children during periods of sleep. Terbutaline (Bricanyl, Astra; 250 $\mu \mathrm{g} / \mathrm{puff}$ ) up to a maximum of four puffs in any four hours, was given through the same delivery system, one puff at a time, for symptomatic treatment.

Children entered the study period only if they had either cough, wheeze, sleep disturbance, or limitation of activity, recorded on at least three days/week for both run-in weeks and if their parents were able to give medications through the Nebuhaler and mask. The study was double blind and children were randomised to receive budesonide $(200 \mu \mathrm{g}$ twice a day) or placebo, in parallel groups for up to six months. They were followed up at six week intervals by the same physician (GJC) and their inhaler technique was checked by a paediatric respiratory nurse specialist $(\mathrm{EW} / \mathrm{CW})$. The dosage of study medication was adjusted by the physician or parent, on the basis of clinical need, between $200-400 \mu \mathrm{g}$ twice a day of budesonide or 1-2 puffs twice a day of placebo. Oral prednisolone (1-2 $\mathrm{mg} / \mathrm{kg} /$ day) was given for acute exacerbations of asthma and nebulised terbutaline 2.5-5 mg up to every four hours was prescribed if symptom control was poor. A record was made of all medication prescribed for the treatment of asthma symptoms. Children were withdrawn if symptom control was poor,

Table 1 Demographic data

\begin{tabular}{|c|c|c|}
\hline Patients & $\begin{array}{l}\text { Budesonide } \\
(n=20)\end{array}$ & $\begin{array}{l}\text { Placebo } \\
(n=20)\end{array}$ \\
\hline \multicolumn{3}{|l|}{ Sex } \\
\hline Male & $11(55)$ & $15(75)$ \\
\hline Female & $9(45)$ & $5(25)$ \\
\hline Mean (SD) age (years) & $1.7(0.6)$ & $1.9(0.5)$ \\
\hline Range & $1 \cdot 0-2 \cdot 7$ & $1 \cdot 2-2 \cdot 6$ \\
\hline Mean (SD) duration of asthma (years) & $1 \cdot 1(0.6)$ & $1.3(0.6)$ \\
\hline Range & $0 \cdot 5-2 \cdot 4$ & $0.5-2 \cdot 5$ \\
\hline Mean (SD) baseline asthma severity (night time cough): & $0.9(0.5)$ & $0.8(0.4)$ \\
\hline Range & $0 \cdot 1-1 \cdot 4$ & $0.0-1 \cdot 9$ \\
\hline \multicolumn{3}{|l|}{ No (\%) with family history of: } \\
\hline Atopy (yes) & $12(60)$ & $15(75)$ \\
\hline Asthma (yes) & $12(60)$ & $11(55)$ \\
\hline Smoking (yes) & $11(55)$ & $9(45)$ \\
\hline Mean (SD) g tobacco/day $\star$ & $17.9(13.2)$ & $20.6(14 \cdot 3)$ \\
\hline Range & $2-40$ & $5-40$ \\
\hline
\end{tabular}

*Tobacco consumption: one cigarette $=1 \mathrm{~g}$; one cheroot $=1 \mathrm{~g}$; one cigar $=5 \mathrm{~g} ; 1 \mathrm{oz}=28 \mathrm{~g}$.

Table 2 Children withdrawn from the study

\begin{tabular}{lcl}
\hline Study group & Days studied & Reason for withdrawal \\
\hline Budesonide & 27 & Non-compliance; did not return for further visits \\
& 47 & Severe asthma complicated by pneumonia \\
& 56 & Mother unable to follow study protocol \\
& 101 & Chronic poorly controlled asthma \\
& 104 & Need for third course of prednisolone \\
& 104 & Poor compliance with the use of delivery system \\
Placebo & 118 & Obvious evidence of fabricated data \\
& 29 & Adverse event: screaming at night \\
& 83 & Need for third course of prednisolone \\
& 94 & Need for third course of prednisolone \\
& 97 & Chronic poorly controlled asthma \\
& 104 & Need for third course of prednisolone \\
& 129 & Need for third course of prednisolone \\
\hline
\end{tabular}

^Insufficient data for analysis. despite the use of back up medication. This was obligatory in those requiring three courses of oral prednisolone. The study had local ethical committee approval and the parents of all children gave informed consent.

\section{ASSESSMENTS}

Parents completed diary cards twice daily. Each morning they recorded the previous night's cough, wheeze, and wheeze on waking using a scale of $0-2 \quad(0=$ no symptoms, $1=$ symptoms present but not troublesome, $2=$ present and troublesome) together with the number of times their own sleep was disturbed by their child's asthma. Each evening they recorded that day's cough and wheeze, using the 0-2 scale, together with any limitation of activity (yes/no). Use of study medication, bronchodilators (aerosol and nebuliser solution; day and night), and time spent caring for their child's asthma (day and night) were also recorded. These assessments, together with a record of the amount of prednisolone received (mg/patient) and the number of prescriptions per patient for asthma, made a total of 14 clinical variables for analysis. Parents of children completing the six month study period were asked if the Nebuhaler and facemask was easy to use, and the physician assessed the benefit of treatment in each of these children.

\section{STATISTICAL ANALYSIS}

The primary efficacy variable in the study was night time cough. We estimated that a difference between treatment group cough scores of 0.5 (scale 0 to 2 ) would be detected, with $80 \%$ power at a $5 \%$ significance level, with 16 patients in each treatment group. Daily diary score card data from the last week of run-in and the last week of treatment were analysed on an intention to treat basis (that is all available diary cards were analysed). The Mann-Whitney $U$ test was used to analyse differences in asthma symptoms, lifestyle assessments, and the use of asthma medication between groups. Differences between groups in the proportion of symptom free days during the study was compared using the $\chi^{2}$ test. The Wilcoxon signed rank sum test was used to make within treatment comparison of lifestyle assessments.

\section{Results}

Of the 49 children entering the run-in, 40 fulfilled the criteria for randomisation into the study, 20 received budesonide and 20 received placebo. The two groups were well matched in terms of demographic data and asthma severity (table 1). Seven children in each group were withdrawn before completion of the full six month study period (table 2 ). Six receiving placebo had poorly controlled asthma, the other was withdrawn within a few weeks of starting the study medication because of screaming attacks at night. These were self limiting and he was later successfully treated 


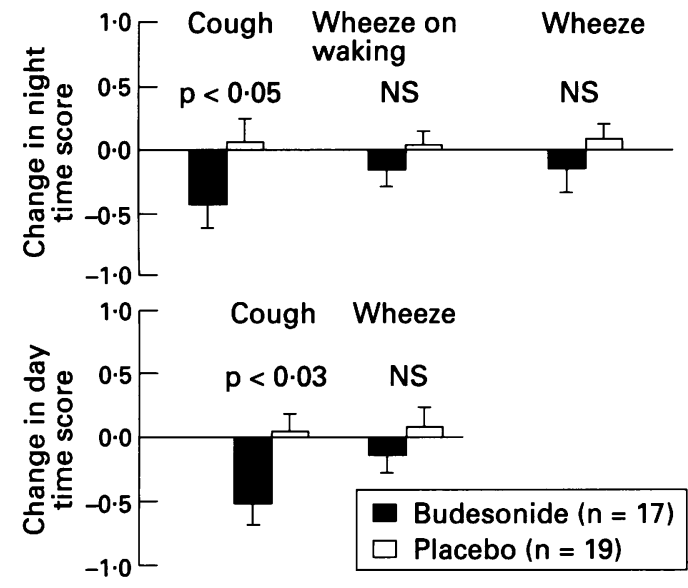

Figure 1 Effects of budesonide or placebo on asthma symptom scores: between treatment comparison of changes from baseline; $N S=$ not significant.

Night time

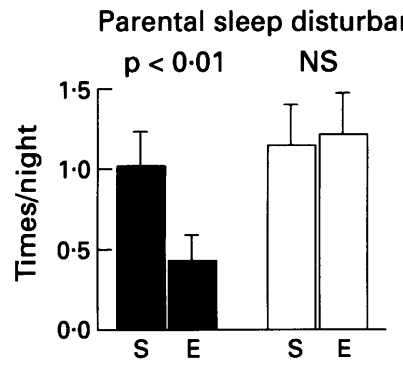

Time spent caring for child's asthma

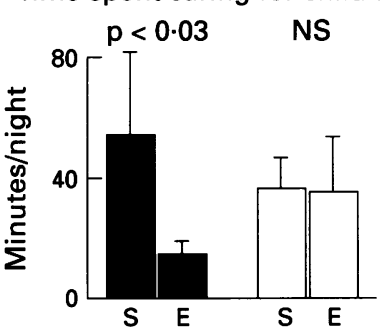

Daytime

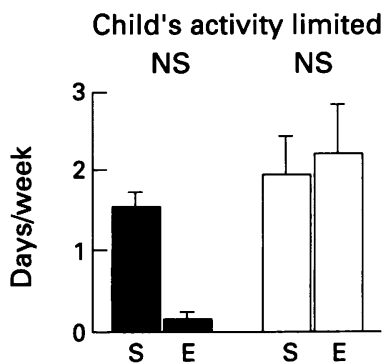

Time spent caring for child's asthme

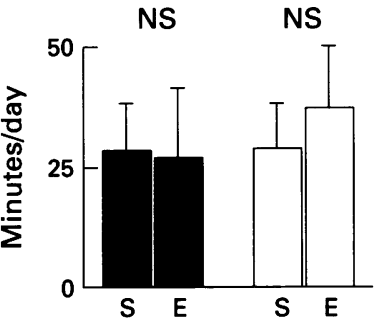

Budesonide $(n=17)$

Placebo $(n=19)$

Figure 2 Effects of budesonide or placebo on lifestyle assessments: within treatment comparison; $S=$ last week of run-in and $E=$ last week of treatment; $N S=$ not significant.

with budesonide through a Nebuhaler and facemask without any further behavioural changes.

In the budesonide group, three did not complete because of poorly controlled asthma and four were withdrawn because parents had difficulties following the study protocol. This left 13 patients in each group completing all six months of the study. Children completing at least five weeks of the study were to be included in the intention to treat analysis. Diary cards were, however, not retrieved from three non-compliant families in the budesonide group, and the diary of the child withdrawn because of screaming contained insufficient data. This left 17 children in the budesonide group and 19 in the placebo group providing data for analysis.

Figure 1 compares the changes (from last week of run-in to last week of treatment) in daily asthma symptom scores after budesonide and placebo treatment. All variables showed improvements favouring the use of budesonide and these were significant for day $(p<0.03)$ and night time $(p<0.05)$ coughing.

Between group comparisons of changes in lifestyle assessments all showed improvements favouring the use of budesonide but were not statistically significant. The number (SD) of times parental sleep was disturbed each night decreased by $0.58(0.81)$ after budesonide compared with $0.06(0.95)$ in the control group $(p=0 \cdot 07)$. Days per week of limited activity fell by $0.71(1.76)$ after budesonide compared with an increase of $0.26(2.26)$ in the control group $(p=0 \cdot 22)$. The time parents spent caring for their child's asthma in the budesonide group decreased by $1.5(51 \cdot 2)$ and $40(89.5)$ minutes per day and night respectively whereas after placebo, daytime caring increased by $8.9(21.3)$ minutes $(\mathrm{p}=0.13)$ and night time caring decreased by $0.9(41.1)$ minutes $(p=0 \cdot 28)$. The within treatment changes in these lifestyle variables are shown in fig 2. The improvements using budesonide were significant for parental sleep disturbance $(p<0.01)$ and time spent attending to their child's asthma during the night $(\mathrm{p}<0.03)$.

Between group comparisons of the use of medication for asthma symptoms are shown in fig 3. Night time bronchodilator use was reduced with budesonide, but increased with placebo treatment $(p<0.05)$. The use of study medication, daytime bronchodilator, oral steroid and asthma medications were also less with budesonide treatment, but these differences were not significant. The proportion of symptom free days in the total study periods was $54 \%$ in children receiving budesonide, and $31 \%$ in children receiving placebo $(p<0.0001)$. Examination of trend data (for day and night time cough and daily bronchodilator usage) showed that improvements with budesonide were evident early in the study. Mean values for symptom scores and bronchodilator usage decreased during the first 11 weeks, and remained at low levels until study completion at 24 weeks. In the placebo group, small improvements in these parameters occurred in the initial weeks of the study, but these were not maintained (data not shown).

Three hospital admissions for asthma exacerbations occurred during budesonide and eight during placebo treatment. In addition, there were five episodes of 'respiratory tract infection' causing worsening of symptoms reported in children receiving budesonide and 10 such episodes occurred in the placebo group. The only drug related adverse event was a case of facial skin rash in the budesonide group. This resolved after the parents were reminded to wipe their child's face after administering the study medication.

Treatment was considered beneficial by the doctor (GJC) in nine out of 13 receiving budesonide and four out of 13 receiving placebo who completed six months of the study ( $p=0 \cdot 115$ using Fisher's exact test). Altogether 25 sets of parents stated that the 


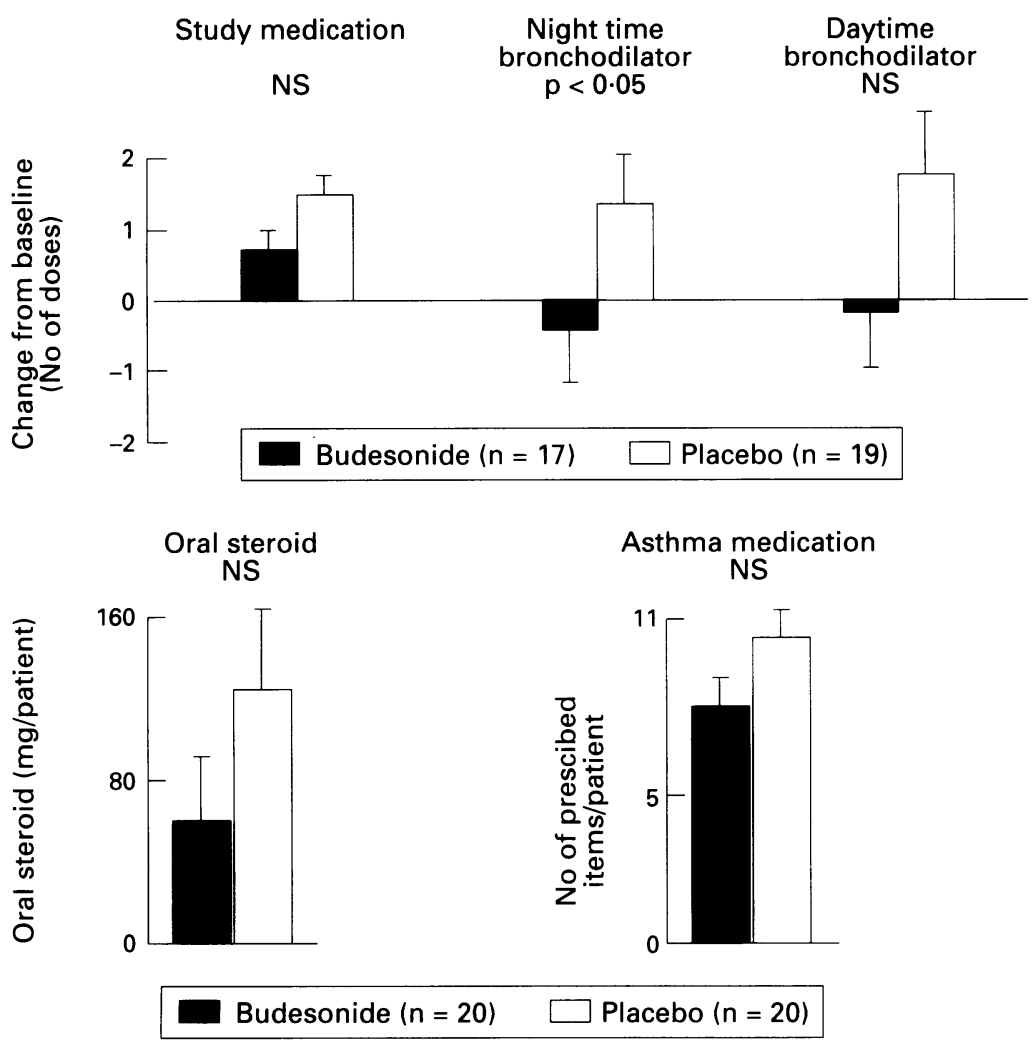

Figure 3 Effect of budesonide or placebo on use of asthma medications: between treatment comparison; $N S=$ not significant.

Nebuhaler and mask was convenient and easy to use.

\section{Discussion}

The study provides evidence for the effective use of budesonide in an age group in whom asthma symptoms are difficult to manage. As well as a decrease in symptoms, sustained improvements in the quality of life of children and parents were also demonstrated. Treatment was unsatisfactory with bronchodilators alone, and nine children had shown no improvement after treatment with nebulised sodium cromoglycate before the study.

Mean scores for the 14 clinical variables assessed in the study consistently favoured the use of budesonide. Despite the overall results, there were individuals who demonstrated the correct use of the delivery system, but who remained clinically unresponsive to treatment.

Although there are many different patterns of asthma, symptoms can be broadly divided into persistent symptoms and acute intermittent exacerbations. The significant increase in symptom free days in children receiving budesonide during the study is good evidence that there were sustainable improvements in the control of persistent symptoms. Acute exacerbations are usually precipitated by viral infections in this age group. ${ }^{16} 17$ All children studied had a history of chronic respiratory symptoms but there were considerable differences in the number of acute attacks of severe asthma. Respiratory infections were probably under reported during the study. They were not easily identifiable in children with persis- tent respiratory illness and trivial symptoms were probably ignored by parents.

Only those causing a marked increase in asthma symptoms and those precipitating the most severe exacerbations of asthma resulting in hospital admission were recorded. As yet, there is no good evidence that regular prophylactic treatment in the preschool age group is useful in preventing asthma attacks, but the decreased number of acute respiratory episodes in the children receiving budesonide suggests that this probably happened in our study.

It is essential when using inhaled steroids, to use the least amount of medication necessary to control symptoms to minimise the possibility of systemic side effects. Dose response studies suggest that clinically important side effects occur when more than $800 \mu \mathrm{g} /$ day of inhaled corticosteroids are used. ${ }^{18}$ The individual sensitivity to steroidal side effects varies between patients and there is some evidence for intrasubject differences in the sensitivity to different steroid compounds. ${ }^{19}$

Recommended administered doses for school age children are not necessarily applicable to younger children inhaling medication through a spacer adapted by the attachment of a facemask. A recent study attempting to quantify drug delivery through this type of delivery system suggested that $9 \cdot 3 \%$ of a $1 \mathrm{mg}$ dose of sodium cromoglycate was delivered using a tidal volume of $50 \mathrm{ml} .{ }^{20}$ Increasing the dead space between the chamber valve and 'face' and agitating the chamber caused large decreases in drug delivery. Hence, the amount of drug reaching the lungs is probably much less. $^{21}$ The administration of inhaled medication in this age group is much more of an art than a science, and it is very likely that delivered doses vary widely between and within children. Our clinical impression is that $200 \mu \mathrm{g}$ twice a day is usually the minimal dose necessary to control symptoms in this group of children with severe asthma.

Measurement of adrenal function is advised in children receiving inhaled steroids. ${ }^{22}$ As yet there is no suitable way of assessing adrenal function in this age group. Single plasma cortisol assays are not very helpful, and 24 hour urine specimens for urinary cortisol measurements are not easy in children who are not yet continent.

Monitoring of growth is also essential, but prolonged periods of measurement are necessary to detect a significant decrease in growth rate. Height velocity during the study was calculated for each patient and were shown to be within normal limits. Knemometry has been used successfully to measure short term changes in growth in this age group $(\mathrm{H}$ Bisgaard, European Pediatric Respiratory Society Meeting, Rome 1991), although the implications of its findings on total height are uncertain. Further research into the clinical importance of the systemic effects of inhaled steroid treatment are needed.

In summary, we have shown that the Nebuhaler and facemask is an acceptable delivery system for long term asthma treatment. 
Symptoms are significantly reduced when it is used to give $400-800 \mu \mathrm{g}$ budesonide/day to children aged 1-3 years with severe asthma.

Thanks are extended to Dr Michael Taylor and Dr Stephen McConchie for their invaluable assistance during this study. McConchie for their invaluable assistance during this study. analysis. Joanne Pell prepared the manuscript. Gary Connett, analysis. Joanne Pell prepared the manuscript. Gary Connett, Royal Alexandra Hospital Rocking Horse Appeal.

1 Sporik R, Holgate ST, Cogswell JJ. Natural history of sthma in childhood - a birth cohort study. Arch Dis Child $1991 ; 66: 1050-3$

2 Chung KF. Role of inflammation in the hyperactivity of the airways in asthma. Thorax 1986; 41: 657-62.

3 Webb MSC, Henry RL, Milner AD. Oral corticosteroids for wheezing attacks under 18 months. Arch Dis Child 1986; wheezing $15-9$.

4 Storr J, Barry W, Barrell E, Hatcher G, Lenney W. Effect of a single dose of prednisolone in acute childhood asthma. Lance 1987; i: 879-82.

5 Tal A, Levy N, Bearman JE. Methylprednisolone therapy for acute asthma in infants and toddlers: a controlled clinical trial. Pediatrics 1990; 86: 350-6.

6 Bisgaard H, Munck SL, Nielsen JP, Petersen W, Ohlsson SV. Inhaled budesonide for treatment of recurrent wheezing in early childhood. Lancet 1990; 336: 649-51.

7 Noble V, Ruggins NR, Everard ML, Milner AD. Inhaled budesonide for chronic wheezing under 18 months of age. Arch Dis Child 1992; 67: 285-8

8 Casaulta Aebischer C, Graf U, Modelska K, Kraemer R. Efficacy of beclomethasone dipropionate (DBP) in combination with salbutamol administered by the babyhaler in wheezy infants. Am Rev Respir Dis 1992; 145: A709.
9 Van Bever HP, Schuddinck L, Wojciechowski M, Stevens WJ. Aerosolized budesonide in asthmatic infants: a double blind study. Pediatr Pulmonol 1990; 9: 177-80.

10 Godfrey S, Avital A, Rosler A, Mandelburg A, Uwyyed K. Nebulised budesonide in severe infantile asthma. Lancet 1987; ii: 851-2.

11 De Jongste JC, Duiverman EJ. Nebulised budesonide in severe childhood asthma. Lancet 1989; i: 1388.

12 Phelan PD, Landau LI, Olinsky A. Asthma: clinical patterns and management. 3rd Ed. Respiratory illnesses in childhood. Oxford: Blackwell Scientific Publications, 1990: 132-8. 13 Anon. Cough and wheeze in asthma: are they interdependent? Lancet 1988; i: 447-8.

14 Thomson AH, Pratt C, Simpson H. Nocturnal cough in asthma. Thorax 1977; 32: 677-83.

15 Hoskyns EW, Thomson A, Decker E, Hutchins A, Simpson H. Effect of controlled release salbutamol on nocturnal cough in asthma. Arch Dis Child 1991; 66: 1209-12.

16 McIntosh K, Ellis EF, Hoffman LS, et al. The association of viral and bacterial respiratory infections with exacerbations of wheezing in young asthmatic children. $f$ Pediatr 1973; 82: 578-90.

17 Mertsola J, Ziegler T, Ruuskanen O, Vanto T, Koivikko A, Halonen $P$. Recurrent wheezy bronchitis and viral respiratory infections. Arch Dis Child 1991; 66: 124-9.

18 Geddes DM. Inhaled corticosteroids: benefits and risks. Thorax 1992; 47: 404-7.

19 Prahl P. Adrenocortical suppression following treatment with beclomethasone and budesonide. Clin Exp Allergy 1991; 21: 145-6.

20 Everard ML, Clark AR, Milner AD. Drug delivery from holding chambers with attached facemask. Arch Dis Child 1992; 67: 580-5.

21 Salmon B, Wilson NM, Silverman M. How much aerosol reaches the lungs of wheezy infants and toddlers? Arch Dis Child 1990; 65: 401-3.

22 Kamada AK, Parks DP, Szefler SJ. Inhaled glucocorticoid therapy in children: how much is safe? Pediatr Pulmonol 1992; 12: 71-7. 